OPEN ACCESS

Edited by:

Giuseppe Sconocchia,

Consiglio Nazionale Delle

Ricerche (CNR), Italy

Reviewed by:

Alessandro Poggi,

Ospedale Policlinico

San Martino, Italy

Loredana Ruggeri,

University of Perugia, Italy

*Correspondence:

Cyrille J. Cohen

cyrille.cohen@biu.ac.il

Specialty section:

This article was submitted

to Cancer Immunity

and Immunotherapy,

a section of the journal

Frontiers in Immunology

Received: 04 June 2017 Accepted: 12 September 2017 Published: 29 September 2017

Citation:

Eisenberg V, Shamalov K, Meir S,

Hoogi S, Sarkar R, Pinker S,

Markel G, Porgador A and Cohen CJ

(2017) Targeting Multiple Tumors

Using T-Cells Engineered to Express

a Natural Cytotoxicity Receptor

2-Based Chimeric Receptor.

Front. Immunol. 8:1212.

doi: 10.3389/fimmu.2017.01212

\section{Targeting Multiple Tumors Using T-Cells Engineered to Express a Natural Cytotoxicity Receptor 2-Based Chimeric Receptor}

\author{
Vasyl Eisenberg ${ }^{1}$, Katerina Shamalov ${ }^{1}$, Shimrit Meir ${ }^{1}$, Shiran Hoogi ${ }^{1}$, Rhitajit Sarkar ${ }^{2,3}$, \\ Shirel Pinker ${ }^{1}$, Gal Markel ${ }^{4}$, Angel Porgador ${ }^{2}$ and Cyrille J. Cohen ${ }^{1 *}$ \\ 'The Laboratory of Tumor Immunology and Immunotherapy, The Mina and Everard Goodman Faculty of Life Sciences, \\ Bar-Ilan University, Ramat Gan, Israel, ${ }^{2}$ Faculty of Health Sciences, The Shraga Segal Department of Microbiology, \\ Immunology and Genetics, The National Institute for Biotechnology in the Negev, Ben-Gurion University of the Negev, \\ Beer Sheva, Israel, ${ }^{3}$ ASAS, Amity University Haryana, Manesar, India, ${ }^{4}$ The Ella Lemelbaum Institute \\ of Immuno-Oncology, Institute of Oncology, Sheba Medical Center, Tel Hashomer, Israel
}

Recent developments in cancer treatment are demonstrating the increasing and powerful potential of immunotherapeutic strategies. In this regard, the adoptive transfer of tumor-specific T-lymphocytes approaches can lead to tumor regression in cancer patients. More recently, the use of T-cells genetically engineered to express cancerspecific receptors such as the anti-CD19 chimeric antigen receptor (CAR) continues to show promise for the treatment of hematological malignancies. Still, there is a crucial need to develop efficient CAR-T cell approaches for the treatment of solid tumors. It has been shown that other lymphocytes such as natural killer (NK) cells can demonstrate potent antitumor function-nonetheless, their use in immunotherapy is rather limited due to difficulties in expanding these cells to therapeutically relevant numbers and to suppression by endogenous inhibitory mechanisms. Cancer recognition by NK cells is partly mediated by molecules termed natural cytotoxicity receptors (NCRs). In the present study, we hypothesize that it is possible to endow T-cells with an NK recognition pattern, providing them with a mean to recognize tumor cells, in a non-MHC restricted way. To test this, we genetically modified human T-cells with different chimeric receptors based on the human NCR2 molecule and then assessed their antitumor activity in vitro and in vivo. Our results show that expression in primary lymphocytes of an NCR2derived CAR, termed s4428z, confers T-cells with the ability to specifically recognize heterogeneous tumors and to mediate tumor cytotoxicity in a mouse model. This study demonstrates the benefit of combining tumor recognition capability of NK cells with T cell effectiveness to improve cancer immunotherapy.

Keywords: NCR2, T-cells engineering, chimeric receptors, adoptive T-cell transfer, T-cell immunotherapy, natural killer cells

\section{INTRODUCTION}

Recent advances in tumor immunotherapy have demonstrated a crucial role for immunosurveillance, as tumor development and progression can be often linked to a failure of the immune system to recognize tumors and to mount an adequate response (1-3). In that regard, natural killer (NK) cells represent a central component of the early anticancer immune response. The latter relies on the 
integrated balance of signals transduced by activating and inhibitory receptors (4-6). Known activating receptors are DNAM-1, NKG2D, and members of the natural cytotoxicity receptors (NCRs) family: NCR1 (NKp46), NCR2 (NKp44), and NCR3 (NKp30) (7). NCR2/Nkp44 was identified by Vitale et al. two decades ago (8) and several studies described its role as an activating receptor important for the function of NK cells (9). NCR2 is involved in tumor recognition and can mediate cytokine and cytotoxic granule secretion $(10,11)$, but some of its isoforms (such as Nkp44-1) bear an ITIM and can dampen NK cell function (11). The ligands of NCRs are often found on viral-infected and tumor cells and as such, NCR molecules can mediate the recognition of a wide range of tumors and their lysis by NK cells (11-20). NCR ligands have also the potential to regulate the receptor function and facilitate tumor escape mechanisms from NK cells $(11,21,22)$, which would lend support to their wide expression on the surface of cancer cells. In particular, newly discovered NCR2 ligands such as PCNA $(11,21)$ and MLL5/NKp44L (23) were showed to be broadly expressed by several tumors and recognized by NK-cells. It would, therefore, be valuable to target them in the context of antitumor immunotherapy and targeted therapies.

Compared to T-cell-based immunotherapy, NK-based treatment of cancer is less prevalent (6), since it has often proven difficult to expand and adequately condition these lymphocytes (24), which may display poor reactivity in vivo (25) and may be rejected when administered in allogeneic settings $(26,27)$. On the other hand, adoptive T-cell transfer can mediate the regression of large solid and hematological malignancies (28-31). In addition to the use of naturally occurring tumor-specific T-cells, we and others showed the feasibility of engineering lymphocytes to express T-cell receptors (TCRs) conferring them novel antitumor activity (32). However, it is important to remember that TCR-based therapy is dependent on the expression of a specific HLA allele that present the targeted epitope (33), thereby restricting its applicability to selected patients. Another type of MHC-independent T-cell specificity engineering approach can be achieved by chimeric antigen receptors (CARs) targeting tumorsurface antigens. CARs are composed of a targeting portion (generally a scFv specific for a defined antigen) and a signaling moiety

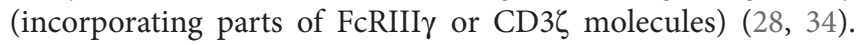
Still, these approaches are often specific for a defined antigen, whose expression may be limited to certain types of cancer, mainly hematological. Importantly, the use of T-cells virally transduced with an anti-CD19 CAR can lead to up to $80-90 \%$ complete regression in ALL patients as well as unprecedented therapeutic efficacy in the treatment of other hematological malignancies (30, $35,36)$. Nonetheless, there is a crucial need to develop efficient CAR-T cell approaches for the treatment of solid tumors (37). To extend the use of chimeric molecules using receptors derived from another type of lymphocytes (NK cells), we and others recently showed that NCR-1 and NCR-3 can be used a targeting moieties in a novel type of CARs derived from NK-cell receptors $(38,39)$ and that these molecules can mediate the recognition of diverse cancers and eradicate tumors in vivo. In the present manuscript, we address the case of NCR2 (also known as NKp44) and show that an optimal NCR2/CD28z chimeric receptor, termed s4428z, mediates antitumor response against multiple tumors in vitro, which translated into high cytokine secretion, superior expression of activation markers, and cytotoxicity in vitro and in vivo using a mouse model of human xenograft tumors.

\section{MATERIALS AND METHODS}

\section{PBMCs and Cell Lines}

PBLs used in this study were from normal donors from the Israeli Blood Bank (Tel-Hashomer, Israel) after obtaining an informed consent. MDA-MB-435 (ATCC/HTB-129) is a previously described malignant breast line derived from metastatic site (pleural effusion) but now considered a melanoma line, DU-145 (ATCC/HTB-81) is a prostate cancer line derived from metastatic site (brain), HeLa (ATCC/CCL-2) is a cervix adenocarcinoma line, and Colo-205 (ATCC/CCL-222) is a colorectal adenocarcinoma cell line derived from metastatic site (ascites). Generation of primary melanoma cultures, $\mathrm{M \# 3}$ and M\#14, was performed as part of clinical adoptive transfer protocols, which were approved by the Israel Ministry of Health (Approval no. 3518/2004, ClinicalTrails.gov Identifier NCT00287131), after obtaining an informed consent from the patients. Packaging line 293GP (expressing GAG and POL) was described (40). Tumor cells were cultured in RPMI (Invitrogen, Carlsbad, CA, USA) or in DMEM (Invitrogen, Carlsbad, CA, USA), both supplemented with 10\% FBS (Biological Industries, Beth Haemek, Israel). Lymphocytes were cultured in BioTarget medium (Biological Industries, Beth Haemek, Israel), 10\% FBS, and 300 IU/ml IL-2. Cells were maintained at $37^{\circ} \mathrm{C}$ and $5 \% \mathrm{CO}_{2}$.

\section{NCR2 Chimeras and Retroviral Constructs}

The cDNA encoding the human NKp44 (NCR2) was amplified from reverse-transcribed mRNA isolated from human NK cells. The different chimeras were created by overlapping PCR $(41,42)$ and their amino acid composition is indicated in Figure 1A. These chimeras as well as a truncated version of CD34 were cloned into the well-characterized retroviral vector backbone pMSGV1 (38), which is a derivative of the murine stem cell virus (MSCV)-based splice-gag vector (pMSGV), and which uses a MSCV long terminal repeat.

\section{Transduction of PBLs}

For virus production, transfection of $2 \times 10^{6} 293 \mathrm{GP}$ cells with $9 \mu \mathrm{g}$ DNA of MSGV1-based retroviral construct and $4.5 \mu \mathrm{g}$ envelop plasmid (VSV-G) was performed using JetPrime transfection reagent (Polyplus, France) (43-45). Retroviral supernatant was collected $36 \mathrm{~h}$ after the DNA transfection. Freshly isolated human PBLs were stimulated for $48 \mathrm{~h}$ in the presence of $50 \mathrm{ng} / \mathrm{ml} \mathrm{OKT3}$ (eBioscience, San Diego, CA, USA) before transduction. Following stimulation, lymphocytes were transduced with retroviral vectors by transfer to non-treated tissue culture dishes (Nunc, Rochester, NY, USA) that had been precoated with RetroNectin (Takara, Japan) and retroviral vectors as previously described (43).

\section{FACS Analysis and Antibodies}

Fluorophore-labeled anti-human CD8, CD137, CD25, and CD34 were purchased from BioLegend (San Diego, CA, USA). 

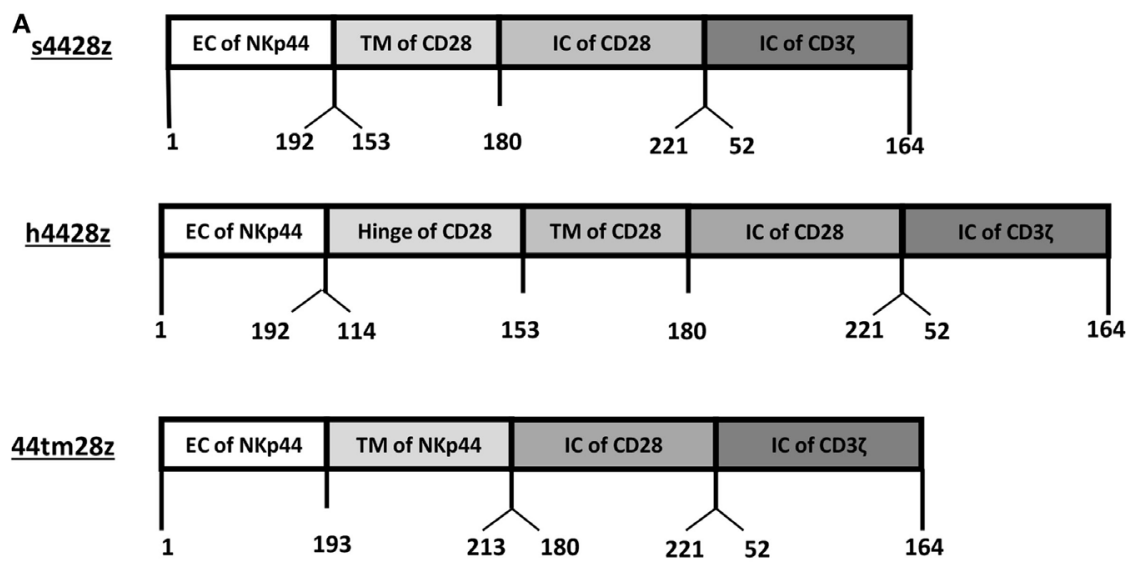

B
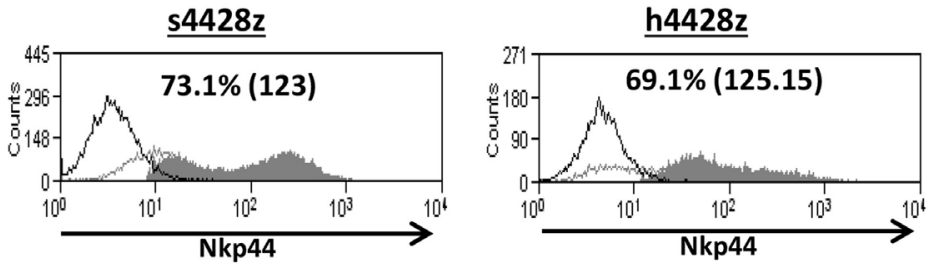

$44 \operatorname{tm} 28 \mathrm{z}$

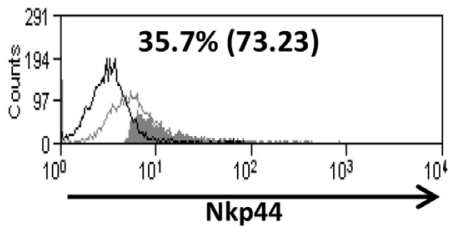

$\underline{\text { CD34 }}$

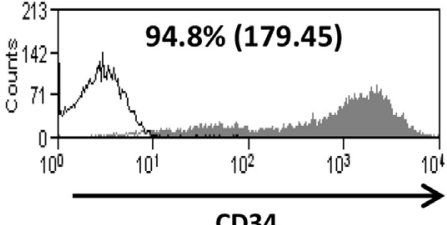

C

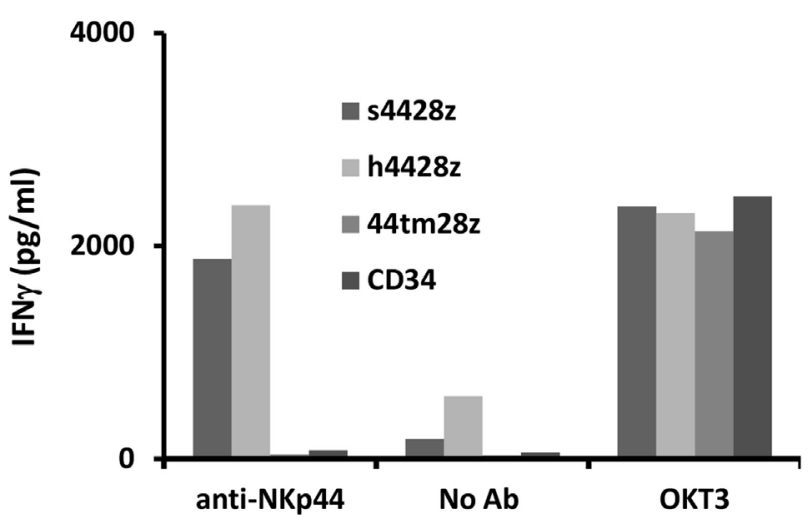

FIGURE 1 | Design and expression of NCR2-based chimeras. (A) Schematic representation of the different NCR-2-based chimeric receptors. The amino acid numbering (based on the original protein) is indicated below each segment. (B) OKT3-stimulated human primary PBLs were transduced with the different versions of NCR2-based chimeric antigen receptor or with truncated CD34 (control gene) as indicated. Transgene expression was assessed by flow cytometry. The dotted line represents the staining of the mock-transduced control. The percentage of positive cells and the MFI (in brackets) are shown. These results are representative of six independent experiments with at least four different donors and the difference between the population transduced with NCR2-chimeric molecules and the control population was found statistically significant $(p<0.05$; calculated using a Student's paired $t$-test). (C) These cells (105) were incubated in a 96-well plate in the presence of plate-bound anti-NCR2 (0.2 $\mu \mathrm{g} /$ well) or OKT3 for $16 \mathrm{~h}$. IFN $\gamma$ secreted in the coculture supernatant was measured by ELISA.

To stain for human NCR2, we used the $3.43 .13 \mathrm{mAb}$ (generously provided by Dr. Marco Colonna, Washington University, St. Louis, MO, USA). Immunofluorescence, analyzed as the relative log fluorescence of live cells, was measured using a
CyAn-ADP flow cytometer (Beckman Coulter, Brea, CA, USA). Approximately $1 \times 10^{4}-1 \times 10^{5}$ cells were analyzed. Cells were stained in a FACS buffer made of PBS, 0.5\% BSA, and 0.02\% sodium azide. 


\section{Cytokine Release Assays}

Lymphocyte cultures were tested for reactivity in cytokine release assays using commercially available ELISA kits for IL-2, IFN $\gamma$, and TNF $\alpha$ (R\&D Systems, Minneapolis, MN, USA). For these assays, $1 \times 10^{5}$ responder cells (T-cells) and $1 \times 10^{5}$ stimulator cells (tumor cells) were incubated in a $0.2-\mathrm{ml}$ culture volume in individual wells of 96-well plates. Stimulator cells and responder cells were cocultured for $18 \mathrm{~h}$. Cytokine secretion was measured in culture supernatants diluted to be in the linear range of the assay. As a control for $\mathrm{T}$ cell activity, we incubated the different T-cell cultures with PMA/ionomycin at a concentration of $50 \mathrm{ng} / \mathrm{ml}$ and $1 \mu \mathrm{M}$, respectively.

\section{Cell Separation}

T-cell populations were separated using a magnetic bead-based approach for negative selection (EasySep TM-StemCell Technologies Inc., Canada).

\section{Cell-Mediated Cytotoxicity Assay}

Target cells were labeled with $2 \mu \mathrm{M}$ CFSE (eBioscience, San Diego, CA, USA) for 6 min and then cocultured with transduced lymphocytes at $37^{\circ} \mathrm{C}$ for $4 \mathrm{~h}$, at different effector:target (E:T) ratios (as indicated in figure legend). After the coculture, propidium iodide (PI) $1 \mu \mathrm{M}$ (Sigma-Aldrich, Israel) was added for assigning the ratio of cell death. Samples were analyzed by flow cytometry.

\section{In Vivo Cytotoxicity Assay-Winn Assay}

6 to 8 weeks year-old NOD-SCD-IL2R-mice (Har-lan, Jerusalem, Israel) were inoculated in the flank with a mixture of $2 \times 10^{6}$ HeLa cells or MDA-MB-435 and $2 \times 10^{6}$ transduced lymphocytes resuspended in $40 \mu$ l Biotarget medium. Tumor size was measured every 3 days using a caliper in a blinded fashion. This study was carried out in accordance with the recommendations of the Bar-Ilan university committee for animal welfare and the Israel Ministry of Health. The protocol was approved by the Bar-Ilan university committee for animal welfare.

\section{Statistical Analysis}

Cytokine secretion, marker upregulation, and cytotoxic activity were compared using a paired Student's $t$-test. Tumor growth slopes were compared using one-way ANOVA. $p<0.05$ was considered statistically significant.

\section{RESULTS}

\section{Evaluation of Different NCR2-Based Chimeric Receptors}

We constructed three different NCR2-chimeric receptors by fusing the extracellular domain of NCR2 (NKp44) to either the transmembrane domain of CD28 (s4428z) or to that of NCR2 (44tm28z) or the hinge of CD28 (h4428z), all of which connected to the CD28 and CD3 $\zeta$ signaling domains (Figure 1A). These were cloned into the MSGV1 retroviral vector and were transduced into OKT3-stimulated primary human PBLs. $48 \mathrm{~h}$ after transduction, we determined the surface expression levels of the different receptors by flow cytometry. We show in Figure 1B that we could detect expression of all the transduced receptors, with s4428z and h4428z demonstrating high expression levels with $73.1 \%(\mathrm{MFI}=123)$ and $69.1 \%(\mathrm{MFI}=125)$, respectively (control gene truncated CD34 showed $94.8 \%$ positive cells). These levels of expression were constant for 30 days following transduction without any selection and the growth and expansion rates of the NCR2-CAR transduced population were comparable to those of the CD34 control T-cells (data not shown).

We then tested the basic function of these receptors by incubating these cells in the presence of plate-bound anti-NCR2 (as a positive control for T-cell activation, we used OKT3 antibody). After $16 \mathrm{~h}$, we harvested the supernatant and measured IFNy concentrations by ELISA. We found that $s 4428 \mathrm{z}$ and $\mathrm{h} 4428 \mathrm{z}$ mediated the highest secretion of IFN $\gamma$ compared to $44 \mathrm{tm} 28 \mathrm{z}$ or the CD34 control (1,876 and 2,393 vs. 40 and $80 \mathrm{pg} / \mathrm{ml}$, respectively). These results correlate with the expression data presented in Figure 1B and suggest that NCR2-based chimeras can function in human T-cells.

\section{s4428z Mediates the Efficient Recognition of Tumors of Different Histologies}

NCR2 ligands are expressed by tumors $(11,21,23)$ and can be recognized by NK cells, though often dampening the function of the latter as we previously showed (21). We decided to test the function of T-cells equipped with the NCR2-CARs described in Figure 1 and to examine to what extent these chimeric receptors would mediate antitumor activity. In parallel, we assessed the expression levels of NCR2-ligands by staining different tumor cell lines with a NCR2/NKp44-Fc molecule and detected significant ligand expression on several tumor lines (MDA-MB-435, HeLa, and DU-145; Figure S1 in Supplementary Material) while the colon carcinoma line Colo205 showed no significant staining.

We assessed the antitumor function mediated by the different NCR2-CARs by setting up a coculture of s4428z-, h4428z-, $44 \mathrm{tm} 28 \mathrm{z}-$, and CD34-transduced primary human T-cells with the aforementioned tumor lines. We detected specific cytokine secretion mediated by the NCR2-chimeras with $\mathrm{s} 4428 \mathrm{z}$ demonstrating the highest of all compared to the CD34-transduced control group (e.g., 4,597 vs. $51 \mathrm{pg} / \mathrm{ml}$ of IFN $\gamma$ in coculture with the cancer line MDA-MB-435; $p<0.05$-Figure 2A). We did not notice any significant cytokine secretion in coculture with CD34 transduced cells or in the presence of Colo205 or normal PBLs (negative controls).

Based on the results presented in Figure 2A, we selected s4428z as our optimal NCR2-based CAR and examined if this receptor could facilitate the secretion of additional cytokines important for T-cell function and antitumor activity such as IL2 and $\mathrm{TNF} \alpha$ (46). As seen in Figure 2B, significant amounts of both aforementioned cytokines were secreted by T-cells expressing s4428z (e.g., 3,381 pg/ml of IL2 and 2,310 pg/ml of TNF $\alpha$ in cocultures with DU145).

We then decided to examine if NCR2-chimeric receptor may cause the upregulation of a T-cell activation marker. s4428z- or truncated CD34-transduced T-cells were cocultured overnight with several cancer cell lines and were analyzed for surface expression of the activation marker 4-1BB (CD137). We observed 
A

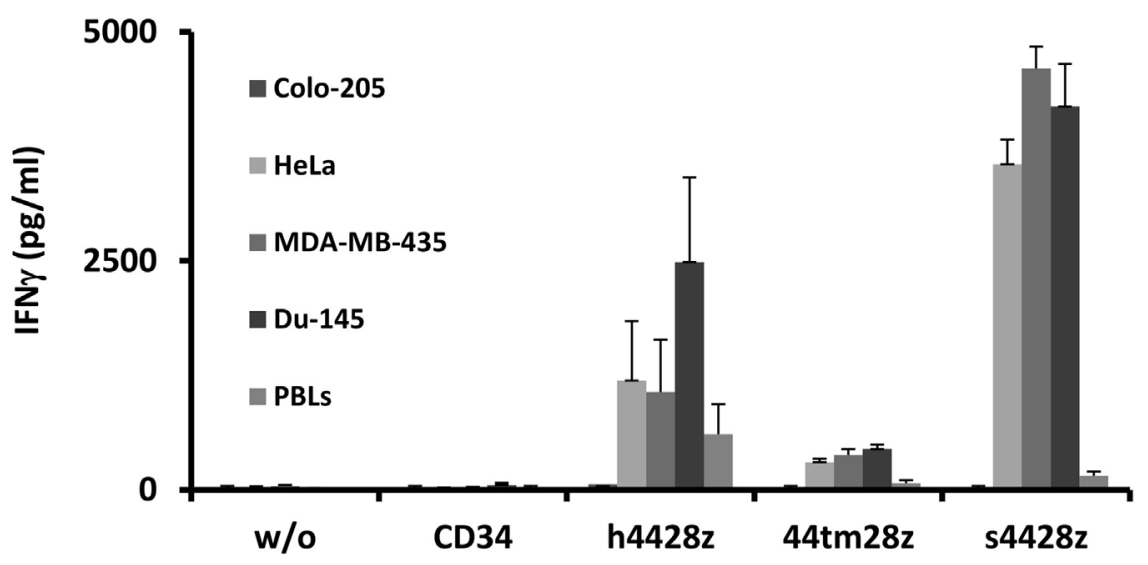

B

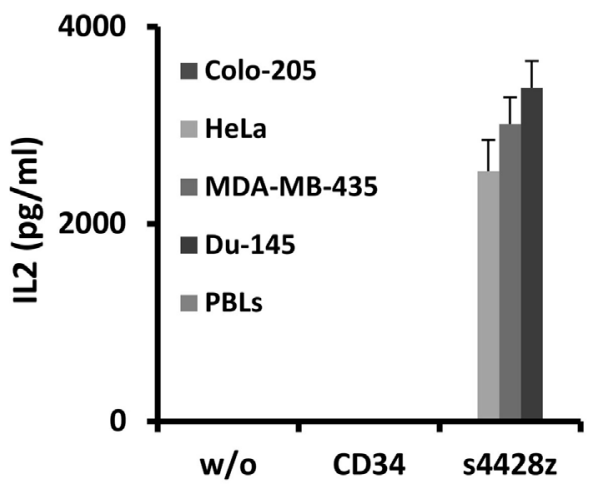

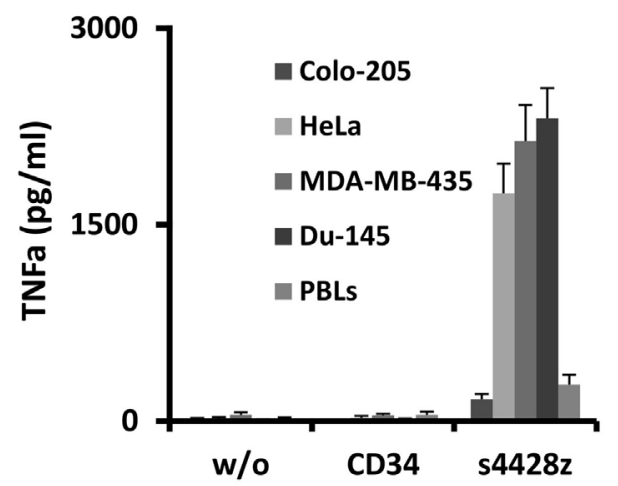
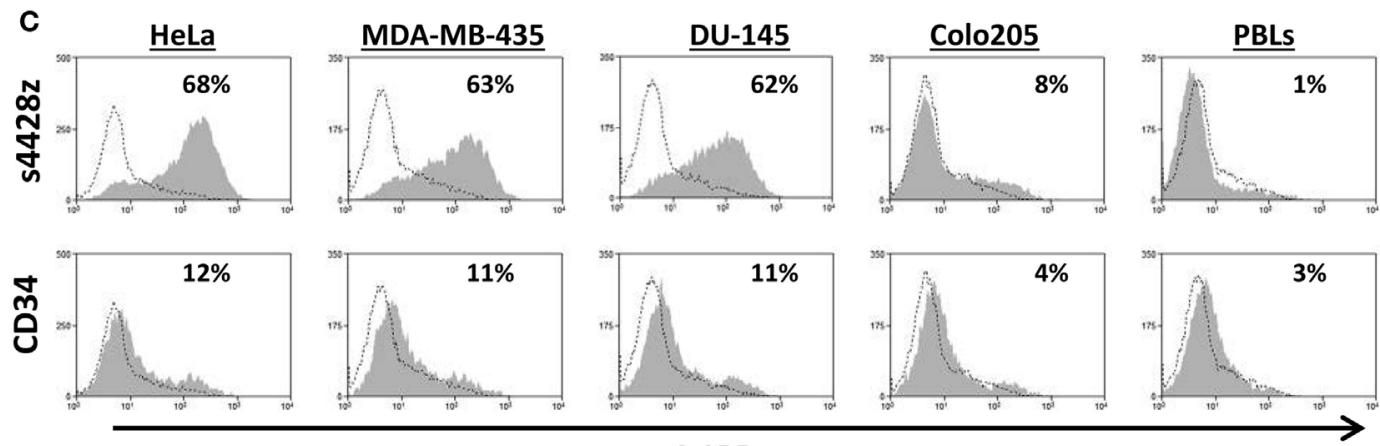

4-1BB

FIGURE 2 | Antitumor function of the NCR2-based chimeras. (A) Human primary lymphocytes were transduced with a retroviral vector encoding either h4428z, s4428z, 44tm28z, and truncated CD34 (control). Transduced T cells were cocultured with different tumor lines or normal PBLs as indicated. IFNy secreted in the coculture supernatant was measured by ELISA. These results are presented as mean + SEM ( $n=5$, with three different donors). (B) s4428z- or CD34-transduced T-cells were cocultured with the indicated target cells and IL2 (right panel) and TNF $\alpha$ (left panel) concentration secreted in the culture supernatant was determined by ELISA. These results are presented as mean + SEM ( $n=5$, with three different donors). (C) Transduced PBLs with either s4428z or CD34 cells were cocultured with tumor lines as indicated and analyzed by flow cytometry for 4-1BB expression gated on the CD8 ${ }^{+}$population. The percentage of positive cells is shown and the dotted line represents the marker staining of the control. These results are representative of three independent experiments with different donors and the difference between s4428z and CD34 was found to be statistically significant ( $p<0.05$, calculated using a Student's paired $t$-test).

that s4428z-engineered $\mathrm{CD}^{+}$cells significantly upregulated the surface expression of $4-1 \mathrm{BB}$ compared to control T-cells (Figure 2C-e.g., $68 \%$ of 4 -1BB-positive cells compared to $12 \%$ for control CD34+). No significant staining was observed in cocultures with the negative control cell line Colo205 or normal PBLs.

Additionally, we assessed whether the $s 4428 \mathrm{z}$ chimeric receptor could mediate the recognition of cells from primary tumor culture. To that end, we used two targets derived from primary melanoma cultures, namely $\mathrm{M} \# 3$ and $\mathrm{M} \# 14$. As controls, we used the DU145 and Colo205 cell lines. As seen in Figure 3A, s4428zequipped T-cells secreted significant amounts of IFN $\gamma$, IL2, and TNF $\alpha$ when cocultured with cells from the primary melanoma culture $\mathrm{M} \# 3$ (e.g., 3,987, 343, and 1,027 $\mathrm{pg} / \mathrm{ml}$, respectively), underscoring the potential clinical relevance of our approach. 

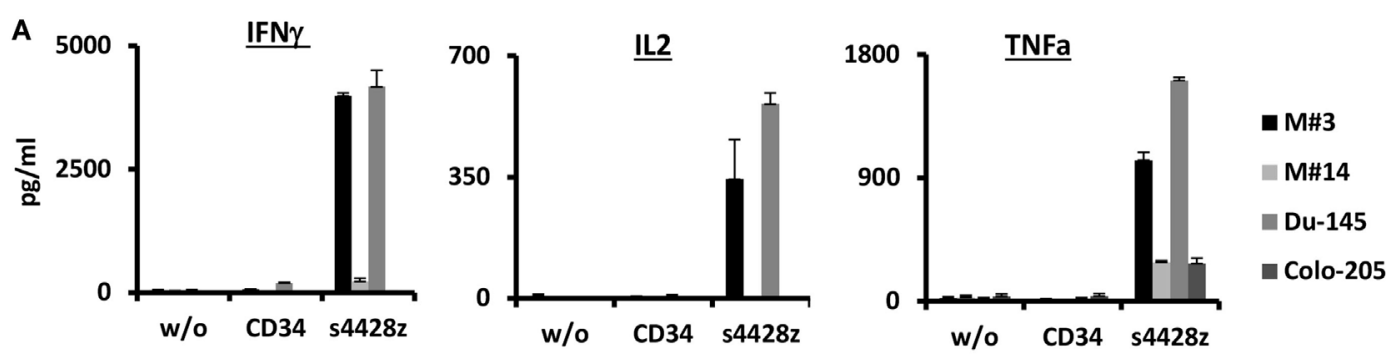

B
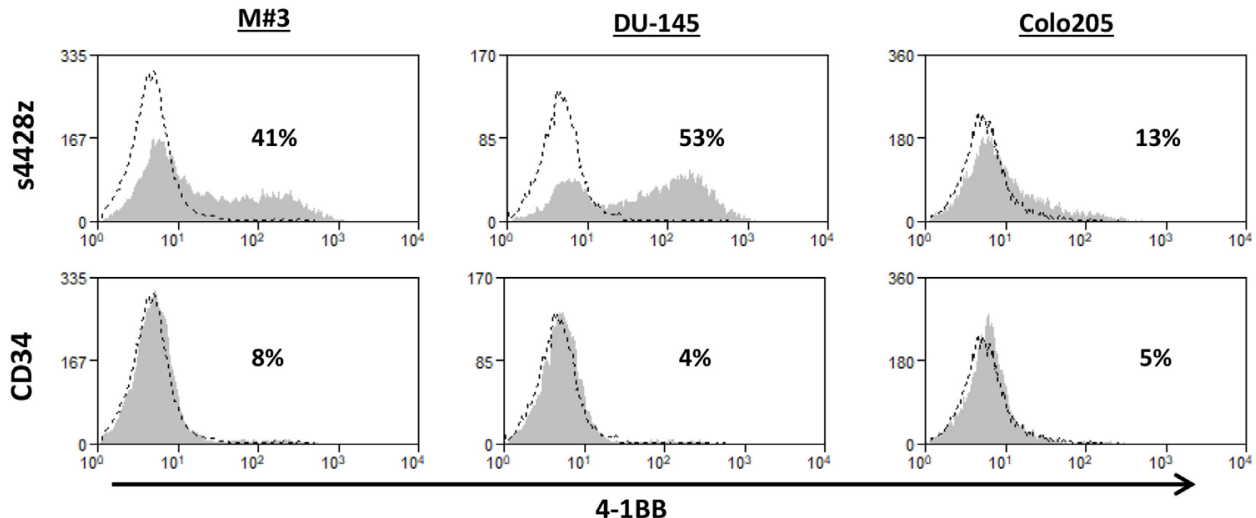

FIGURE 3 | Function of the s4428z chimera against cells derived from primary melanoma culture. (A) Human primary lymphocytes were transduced with a retroviral vector encoding s4428z and truncated CD34 (control). Transduced T cells were cocultured with cells derived from primary melanoma cultures (M\#3 and M\#14) as well as two control tumor lines (DU145 positive and Colo205 negative controls). IFN $\gamma$, IL2, and TNF $\alpha$ secreted in the coculture supernatant was measured by ELISA. These results are presented as mean + SEM ( $n=3$, with two different donors). (B) Transduced PBLs with either s4428z or CD34 cells were cocultured with the indicated targets and analyzed by flow cytometry for 4-1BB expression gated on the CD8 ${ }^{+}$population. The percentage of positive cells is shown and the dotted line represents the marker staining of the control. These results are representative of three independent experiments with different donors and the difference between s4428z and CD34 was found to be statistically significant $(p<0.05$, calculated using a Student's paired $t$-test).

Moreover, we observed an increased surface expression of the T-cell activation marker $4-1 \mathrm{BB}$ by $\mathrm{s} 4428 \mathrm{z}$-expressing T-cells cocultured with M\#3 (Figure 3B-e.g., 41\% of 4-1BB-positive cells compared to $8 \%$ for control CD $34^{+}$transduced T-cells). Overall, these results indicate that $s 4428 \mathrm{z}$ can mediate the efficient recognition of tumors of multiple histologies.

\section{s4428z Chimeric Receptor Can Facilitate the Activation CD4 ${ }^{+}$ T-Lymphocytes}

As widely reported, $\mathrm{CD} 4^{+} \mathrm{T}$-cell responses are important to the coordination of immune responses (47-49). In contrast to TCRs, CARs (including the aforementioned $\mathrm{s} 4428 \mathrm{z}$ chimeric receptor) may function in a non-MHC-restricted manner. Therefore, we surmised that the latter should exert an immunostimulatory effect on $\mathrm{CD}^{+} \mathrm{T}$ cells. To test this, we purified human primary $\mathrm{CD} 4^{+}$ cells and transduced them with either $\mathrm{s} 4428 \mathrm{z}$ or truncated CD34. We cocultured these cells with different cancer lines and analyzed IL-2 and IFN $\gamma$ secretion as well as activation marker upregulation. As expected, s4428z mediated a significant cytokine secretion by transduced lymphocytes compared to the CD34 control (e.g., 23,078 pg/ml of IFN $\gamma$ and 2,818 pg/ml of IL2 in cocultures with the DU145 target cell line-Figure 4A). Moreover, s4428z caused a specific upregulation of the activation marker CD25 in $\mathrm{CD}^{+}{ }^{+} \mathrm{T}$-cells as seen in Figure 4B; e.g., $34.6 \%$ of $\mathrm{CD} 25^{+}$cells compared to $1 \%$ for the CD34 control in coculture with the MDA-MB-435 cell line. These results show that $s 4428 \mathrm{z}$ can provide $\mathrm{CD} 4^{+} \mathrm{T}$-lymphocytes with direct antitumor functionality.

\section{s4428z Mediates Cytotoxicity In Vitro and In Vivo}

We decided to examine the potential of $\mathrm{s} 4428 \mathrm{z}$ in mediating T-cell cytotoxic activity and set up cell-mediated cytotoxicity assays; we labeled tumor cells with CFSE and cocultured those with $\mathrm{CD}^{+}$ T-cells transduced to express the $s 4428 \mathrm{z}$ construct. We analyzed cytotoxicity by flow cytometry after adding to those cocultures PI, which stained dead cells and we observed a statistically significant antitumor cytotoxic activity mediated by s4428z-transduced lymphocytes as shown by the extent of PI-positive population (Figure $5 \mathrm{~A}-58 \%$ for $\mathrm{s} 4428 \mathrm{z}$ vs. $18 \%$ for CD34-transuced cells using the DU145 target cell-line at E:T 5:1; $p<0.05)$.

Finally, the antitumor cytotoxic activity of $s 4428 \mathrm{z}$-transduced T-cells was further confirmed in vivo assays; we tested the ability of s4428z-transduced T-cells to mediate enhanced tumor killing activity in Winn assays using a xenograft model of human tumor. NS-G mice were inoculated with a mixture of either HeLa or MDA-MB-435 cells and s4428z- or CD34-transduced T lymphocytes (at E:T of 1:1), and we measured tumor development in the 

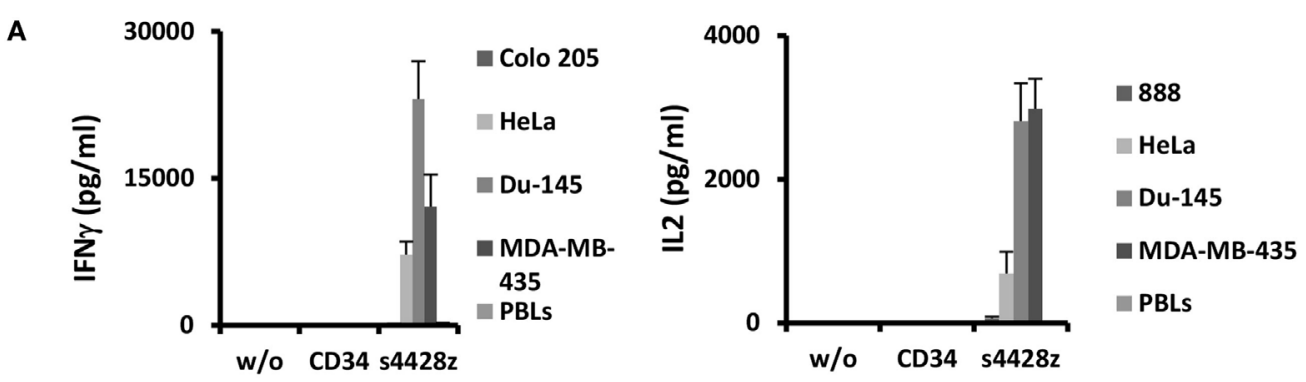

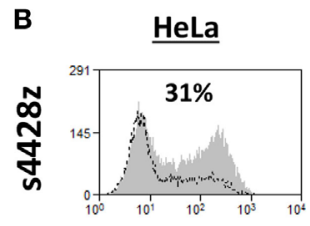

MDA-MB-435

DU-145
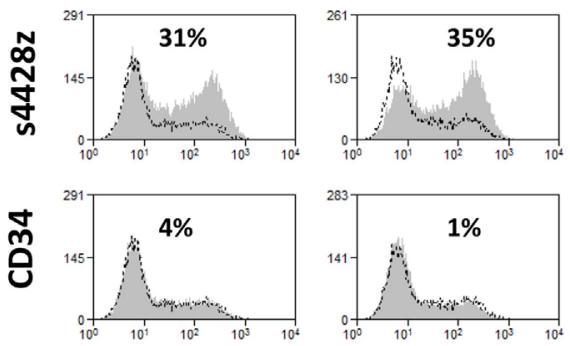

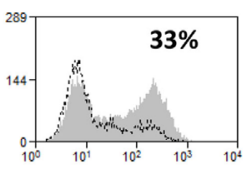

Colo205

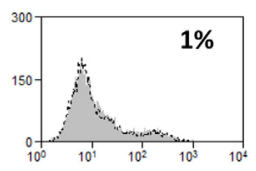

PBLs
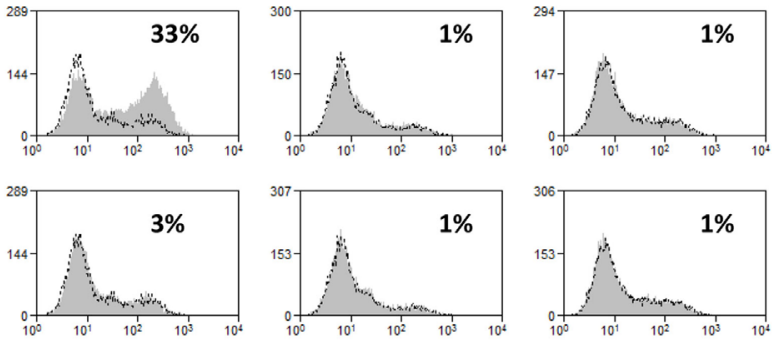

CD25

FIGURE 4 | s4428z function in CD4+ cells. (A) s4428z- or CD34-transduced purified CD4+ cells were cocultured with different tumor lines or not as indicated. IFN $\gamma$ (right panel) and IL2 (left panel) secreted in the coculture supernatant was measured by ELISA. These results are representative of three independent experiments, performed with different donors and the difference between the $s 4428 \mathrm{z}$ and CD34 populations was found statistically significant $(p<0.05$, calculated using a Student's paired $t$-test). (B) CD4+-transduced T cells were analyzed by flow cytometry for CD25 expression following overnight coculture with target cells as indicated. The percentage of positive cells is shown and the dotted line represents the marker staining of the control. These results are representative of three independent experiments and the difference between S4428z and CD34 was found to be statistically significant $(p<0.05$, calculated using a Student's paired $t$-test).

following weeks. We show in Figure 5B that the s4428z-treated group displayed a statistically significant reduced tumor growth when compared to the CD34 or untreated (control) group for both tumor targets $(p<0.05)$. We thus conclude that the s $4428 \mathrm{z}$ chimeric receptor is capable of mediating antitumor cytotoxicity both in in vitro and in vivo settings.

\section{DISCUSSION}

Immunotherapy treatments have achieved widespread clinical benefits, proving that the immune system is capable of containing and eradicating tumors. In that regard, the use of CAR therapy is becoming a promising strategy for the treatment of cancer (28), mainly applied to hematological malignancies. Herein, in order to develop novel CARs targeting solid malignancies, we explored the possibility of utilizing a member of the natural cytotoxicity receptor family, NCR2, as a targeting moiety to redirect T-lymphocytes specificity toward a panel of solid tumors, taking advantage of NCR2-ligand expression by cancer cells.

We designed three NCR2-based chimeric receptors and evaluated their function in primary human T-cells. We mainly modified either the hinge or transmembrane domains as these can considerably influence CAR function (44). Indeed, as seen in Figure 1B, the NCR2-based CAR incorporating the original hinge/transmembrane region of NCR2 showed a decreased stability, which apparently led to poor function (Figure 1C).
It appears, therefore, that CD28 transmembrane region is important for NCR2-CAR function. Additionally, our results with the different NCR2/CD28 CARs studied herein emphasize the need to evaluate both the expression and the function of chimeric receptors empirically as previously recommended (42, 50, 51). Also, since several studies showed that the inclusion of other co-stimulatory domains [derived for example from CD27 (52), 41BB (53-55), OX40 (56)] may result in additional CAR functionality, further optimization is warranted.

Following basic characterization, $\mathrm{s} 4428 \mathrm{z}$ was selected as our optimal construct and was expressed in human primary T-cells to enable the targeting of tumors of multiple histologies. An added value to this approach would be the possibility to target several malignancies using the same non-MHC restricted receptor $(38,39$, 57-60). Such a CAR could, therefore, represent a valuable and available reagent for the immunotherapeutic treatment of numerous cancer patients. Unlike in the case of NK cells in which NCR2-ligands can induce immunosuppressive signals (11), s4428z-expressing T-cells can derive benefit from high NCR2ligand expression. Yet, since the exhaustive identification of NCR2 cellular ligands is still an ongoing task (7), one should consider including a suicide gene in the therapeutic vector configuration when envisaging clinical applications (61). Nonetheless, our in vivo assays (Figure 5B) provide preliminary and promising preclinical data exemplifying the therapeutic potential of the present approach. 

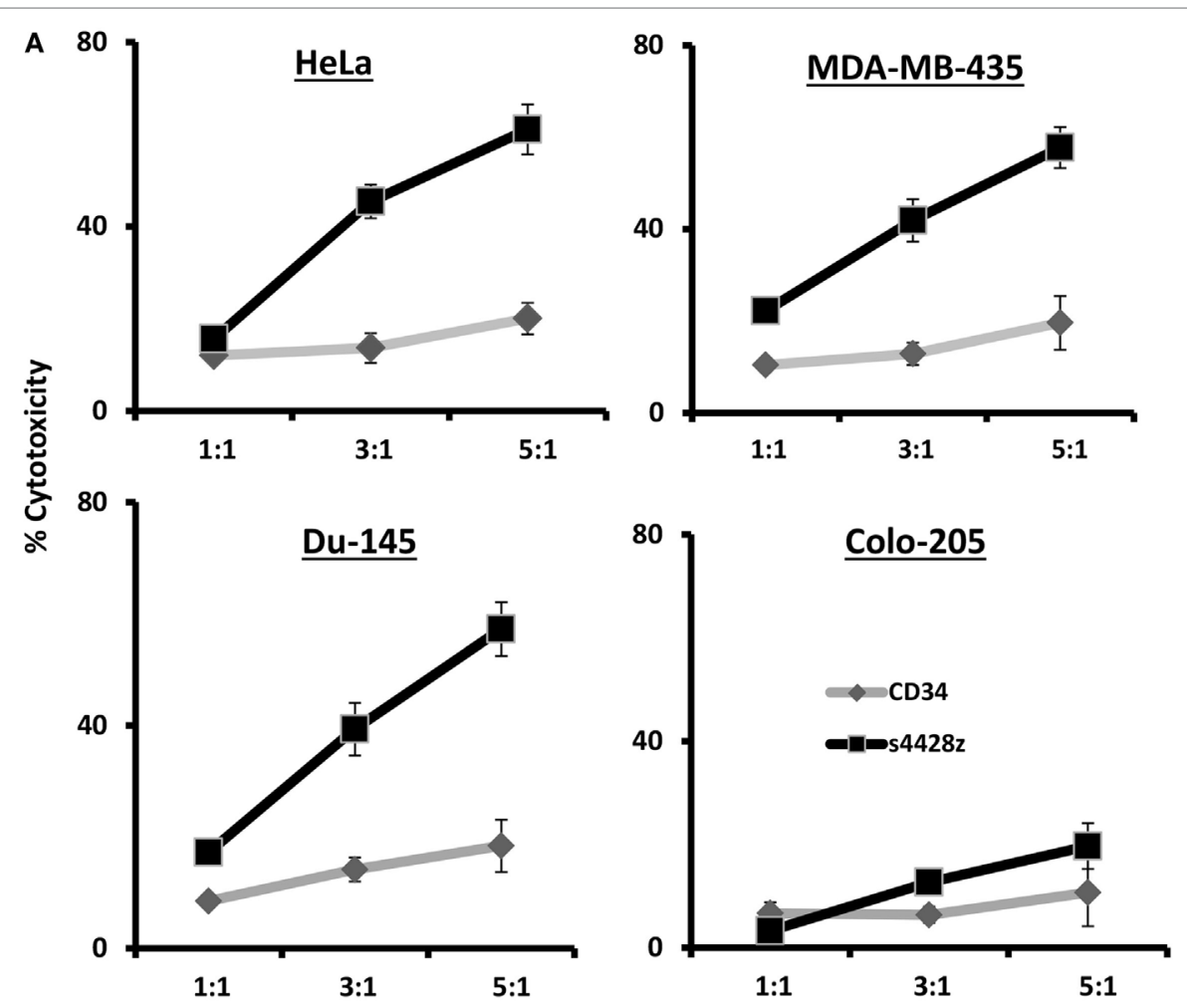

\section{E:T ratio}

B

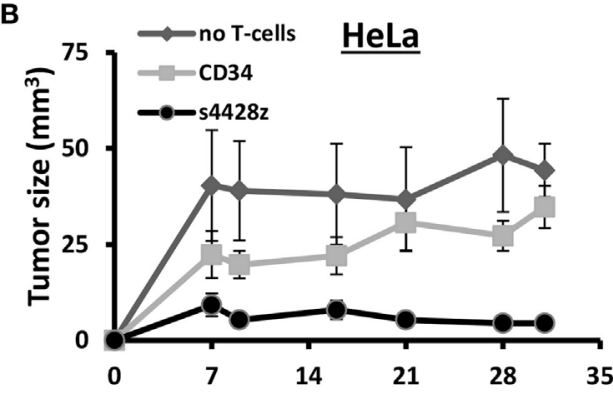

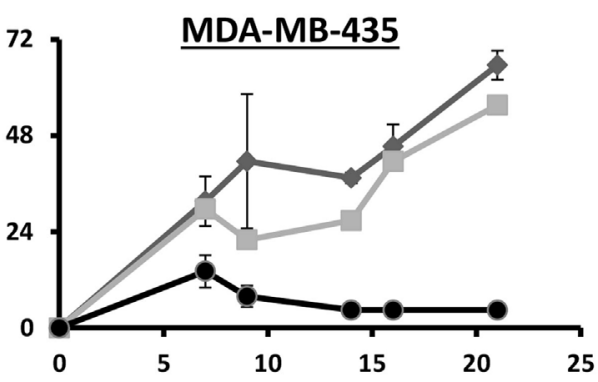

Day after injection

FIGURE 5 | s4428z mediates antitumor cytotoxic activity. (A) s4428z- or CD34-transduced cells were cocultured with the indicated CFSE-labeled tumor cells at the indicated (E:T) ratios. After $4 \mathrm{~h}$, propidium iodide was added and the cells were analyzed by flow cytometry. Cytotoxicity was calculated based on the CFSE+/PI+ population out of the total CFSE+ population. These results are presented as mean + SEM of three independent experiments with three different donors and the difference between the $s 4428 z$ and CD34 populations was found statistically significant ( $p<0.05$, calculated using a Student's paired $t$-test). (B) Winn assay. NS-G mice were inoculated with HeLa or MDA-MB-435 cells and transduced lymphocytes (either s4428z, CD34-control or not as indicated) in the flank. Tumor growth was measured in a blinded fashion using a caliper and calculated using the following formula: $\left(D \times d^{2}\right) \times \Pi 6$, where $D$ is the largest tumor diameter and $d$ its perpendicular one. These results are representative of two experiments (four to five mice in the treated groups) and are shown for the different time points as mean + SEM. The difference between the s4428z and CD34-treated groups was found statistically significant $(p<0.05)$.

We show in Figure 4 that s4428z can mediate antitumor function when expressed in $\mathrm{CD} 4^{+}$human primary T-lymphocytes. The option of recruiting $\mathrm{CD} 4^{+} \mathrm{T}$ lymphocytes in the microenvironment is attractive as they may provide some local support to sustain the CTL antitumor response (62-64). Nevertheless, such option should be ultimately applied only to certain helper T-cell populations such as Th1 but not Th17 or Tregs as these bear an uncertain role in tumor promotion via inflammation or may dampen antitumor immunity, respectively. It has been suggested that NCR expression may promote the rapid response to pathogens in a non-antigen-specific manner (65), and we and others have shown that NCR2 is important to the recognition and elimination of certain bacterial and viral infections $(19,66)$. Therefore, the extension of NCR-based CAR to target the treatment of such infections should be evaluated. 
In conclusion, we have designed in the present study an optimized NCR2-based CAR capable of targeting multiple malignancies. This study demonstrates the benefit of combining tumor recognition capability of NK cells with T cell effectiveness to improve cancer immunotherapy, and we trust the kind of approach can improve the versatility of treatments based on the adoptive transfer of genetically engineered T-cells.

\section{ETHICS STATEMENT}

This study was carried out in accordance with the recommendations of the Bar-Ilan university committee for animal welfare and the Israel Ministry of Health. The protocol was approved by the Bar-Ilan university committee for animal welfare. Generation of

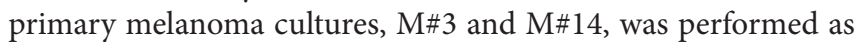
part of clinical adoptive transfer protocols, which were approved by the Israel Ministry of Health (Approval no. 3518/2004, ClinicalTrails.gov Identifier NCT00287131), after obtaining an informed consent from the patients.

\section{AUTHOR CONTRIBUTIONS}

VE performed the experiments, analyzed the data, wrote the manuscript, and prepared the figures. $\mathrm{KS}, \mathrm{SM}, \mathrm{SH}$, and $\mathrm{RS}$

\section{REFERENCES}

1. Swann JB, Smyth MJ. Immune surveillance of tumors. J Clin Invest (2007) 117:1137-46. doi:10.1172/JCI31405

2. Hanahan D, Weinberg RA. Hallmarks of cancer: the next generation. Cell (2011) 144:646-74. doi:10.1016/j.cell.2011.02.013

3. Corthay A. Does the immune system naturally protect against cancer? Front Immunol (2014) 5:197. doi:10.3389/fimmu.2014.00197

4. Vivier E, Tomasello E, Baratin M, Walzer T, Ugolini S. Functions of natural killer cells. Nat Immunol (2008) 9:503-10. doi:10.1038/ni1582

5. Purdy AK, Campbell KS. Natural killer cells and cancer: regulation by the killer cell Ig-like receptors (KIR). Cancer Biol Ther (2009) 8:2211-20. doi:10.4161/ cbt.8.23.10455

6. Pahl J, Cerwenka A. Tricking the balance: NK cells in anti-cancer immunity. Immunobiology (2017) 222:11-20. doi:10.1016/j.imbio.2015.07.012

7. Koch J, Steinle A, Watzl C, Mandelboim O. Activating natural cytotoxicity receptors of natural killer cells in cancer and infection. Trends Immunol (2013) 34:182-91. doi:10.1016/j.it.2013.01.003

8. Vitale M, Bottino C, Sivori S, Sanseverino L, Castriconi R, Marcenaro E, et al. NKp44, a novel triggering surface molecule specifically expressed by activated natural killer cells, is involved in non-major histocompatibility complexrestricted tumor cell lysis. JExp Med (1998) 187:2065-72. doi:10.1084/ jem.187.12.2065

9. Horton NC, Mathew PA. NKp44 and natural cytotoxicity receptors as damage-associated molecular pattern recognition receptors. Front Immunol (2015) 6:31. doi:10.3389/fimmu.2015.00031

10. Cantoni C, Bottino C, Vitale M, Pessino A, Augugliaro R, Malaspina A, et al. NKp44, a triggering receptor involved in tumor cell lysis by activated human natural killer cells, is a novel member of the immunoglobulin superfamily. J Exp Med (1999) 189:787-96. doi:10.1084/jem.189.5.787

11. Rosental B, Brusilovsky M, Hadad U, Oz D, Appel MY, Afergan F, et al. Proliferating cell nuclear antigen is a novel inhibitory ligand for the natural cytotoxicity receptor NKp44. J Immunol (2011) 187:5693-702. doi:10.4049/ jimmunol.1102267

12. Mandelboim O, Lieberman N, Lev M, Paul L, Arnon TI, Bushkin Y, et al. Recognition of haemagglutinins on virus-infected cells by NKp46 activates lysis by human NK cells. Nature (2001) 409:1055-60. doi:10.1038/35059110 performed experiments and analyzed data. SP performed experiments. GM provided vital reagents and designed experiments. AP analyzed data. CC designed experiments, analyzed data, and wrote the manuscript.

\section{ACKNOWLEDGMENTS}

The authors wish to thank Dr. Miriam Horowitz-Fried for technical help, Dr. Levi-Drummer for statistical analysis, Avrille Goldreich for English editing, and Dr. Neta Moskovits and Mr. Meir Huta for animal care. The authors also thank the Cohen's lab team for helpful discussions.

\section{FUNDING}

This work was supported by the Israel Cancer Association and the Israel Science Foundation (1422/15).

\section{SUPPLEMENTARY MATERIAL}

The Supplementary Material for this article can be found online at http://journal.frontiersin.org/article/10.3389/fimmu.2017.01212/ full\#supplementary-material.

13. Glasner A, Ghadially H, Gur C, Stanietsky N, Tsukerman P, Enk J, et al. Recognition and prevention of tumor metastasis by the NK receptor NKp46/NCR1. J Immunol (2012) 188:2509-15. doi:10.4049/jimmunol.1102461

14. Lakshmikanth T, Burke S, Ali TH, Kimpfler S, Ursini F, Ruggeri L, et al. NCRs and DNAM-1 mediate NK cell recognition and lysis of human and mouse melanoma cell lines in vitro and in vivo. J Clin Invest (2009) 119:1251-63. doi:10.1172/JCI36022

15. Sivori S, Parolini S, Marcenaro E, Castriconi R, Pende D, Millo R, et al. Involvement of natural cytotoxicity receptors in human natural killer cellmediated lysis of neuroblastoma and glioblastoma cell lines. J Neuroimmunol (2000) 107:220-5. doi:10.1016/S0165-5728(00)00221-6

16. Nowbakht P, Ionescu MC, Rohner A, Kalberer CP, Rossy E, Mori L, et al. Ligands for natural killer cell-activating receptors are expressed upon the maturation of normal myelomonocytic cells but at low levels in acute myeloid leukemias. Blood (2005) 105:3615-22. doi:10.1182/blood-2004-07-2585

17. Elboim M, Gazit R, Gur C, Ghadially H, Betser-Cohen G, Mandelboim O. Tumor immunoediting by NKp46. J Immunol (2010) 184:5637-44. doi:10.4049/ jimmunol.0901644

18. Wai LE, Garcia JA, Martinez OM, Krams SM. Distinct roles for the NK cellactivating receptors in mediating interactions with dendritic cells and tumor cells. J Immunol (2011) 186:222-9. doi:10.4049/jimmunol.1002597

19. Arnon TI, Lev M, Katz G, Chernobrov Y, Porgador A, Mandelboim O. Recognition of viral hemagglutinins by NKp44 but not by NKp30. Eur J Immunol (2001) 31:2680-9. doi:10.1002/1521-4141(200109)31:9<2680:: AID-IMMU2680>3.0.CO;2-A

20. Hershkovitz O, Jivov S, Bloushtain N, Zilka A, Landau G, Bar-Ilan A, et al. Characterization of the recognition of tumor cells by the natural cytotoxicity receptor, NKp44. Biochemistry (2007) 46:7426-36. doi:10.1021/ bi7000455

21. Rosental B, Hadad U, Brusilovsky M, Campbell KS, Porgador A. A novel mechanism for cancer cells to evade immune attack by NK cells: the interaction between NKp44 and proliferating cell nuclear antigen. Oncoimmunology (2012) 1:572-4. doi:10.4161/onci.19366

22. Brusilovsky M, Radinsky O, Cohen L, Yossef R, Shemesh A, Braiman A, et al. Regulation of natural cytotoxicity receptors by heparan sulfate proteoglycans in -cis: a lesson from NKp44. Eur J Immunol (2015) 45:1180-91. doi:10.1002/ eji.201445177 
23. Baychelier F, Sennepin A, Ermonval M, Dorgham K, Debre P, Vieillard V. Identification of a cellular ligand for the natural cytotoxicity receptor NKp44. Blood (2013) 122:2935-42. doi:10.1182/blood-2013-03-489054

24. Ni J, Miller M, Stojanovic A, Cerwenka A. Toward the next generation of NK cell-based adoptive cancer immunotherapy. Oncoimmunology (2013) 2:e23811. doi:10.4161/onci.23811

25. Parkhurst MR, Riley JP, Dudley ME, Rosenberg SA. Adoptive transfer of autologous natural killer cells leads to high levels of circulating natural killer cells but does not mediate tumor regression. Clin Cancer Res (2011) 17:6287-97. doi:10.1158/1078-0432.CCR-11-1347

26. Lundqvist A, McCoy JP, Samsel L, Childs R. Reduction of GVHD and enhanced antitumor effects after adoptive infusion of alloreactive Ly49-mismatched NK cells from MHC-matched donors. Blood (2007) 109:3603-6. doi:10.1182/ blood-2006-05-024315

27. Cheng M, Chen Y, Xiao W, Sun R, Tian Z. NK cell-based immunotherapy for malignant diseases. Cell Mol Immunol (2013) 10:230-52. doi:10.1038/ cmi.2013.10

28. Merhavi-Shoham E, Haga-Friedman A, Cohen CJ. Genetically modulating T-cell function to target cancer. Semin Cancer Biol (2012) 22:14-22. doi:10.1016/j.semcancer.2011.12.006

29. Restifo NP, Dudley ME, Rosenberg SA. Adoptive immunotherapy for cancer: harnessing the T cell response. Nat Rev Immunol (2012) 12:269-81. doi:10.1038/nri3191

30. Feldman SA, Assadipour Y, Kriley I, Goff SL, Rosenberg SA. Adoptive cell therapy - tumor-infiltrating lymphocytes, T-cell receptors, and chimeric antigen receptors. Semin Oncol (2015) 42:626-39. doi:10.1053/j. seminoncol.2015.05.005

31. Galluzzi L, Vacchelli E, Bravo-San Pedro JM, Buque A, Senovilla L, Baracco EE, et al. Classification of current anticancer immunotherapies. Oncotarget (2014) 5:12472-508. doi:10.18632/oncotarget.2998

32. Daniel-Meshulam I, Yảacobi S, Ankri C, Cohen CJ. How (specific) would like your T-cells today? Generating T-cell therapeutic function through TCRgene transfer. Front Immunol (2012) 3:186. doi:10.3389/fimmu.2012.00186

33. Cohen CJ, Denkberg G, Segal D, Reiter Y. Generation of recombinant immunotoxins for specific targeting of tumor-related peptides presented by MHC molecules. Methods Mol Biol (2003) 207:269-82. doi:10.1385/ 1-59259-334-8:269

34. Gross G, Eshhar Z. Therapeutic potential of $\mathrm{T}$ cell chimeric antigen receptors (CARs) in cancer treatment: counteracting off-tumor toxicities for safe CAR T cell therapy. Annu Rev Pharmacol Toxicol (2016) 56:59-83. doi:10.1146/ annurev-pharmtox-010814-124844

35. Geldres C, Savoldo B, Dotti G. Chimeric antigen receptor-redirected $\mathrm{T}$ cells return to the bench. Semin Immunol (2016) 28:3-9. doi:10.1016/ j.smim.2015.12.001

36. Chmielewski M, Hombach AA, Abken H. Of CARs and TRUCKs: chimeric antigen receptor (CAR) $\mathrm{T}$ cells engineered with an inducible cytokine to modulate the tumor stroma. Immunol Rev (2014) 257:83-90. doi:10.1111/ imr. 12125

37. Han S, Latchoumanin O, Wu G, Zhou G, Hebbard L, George J, et al. Recent clinical trials utilizing chimeric antigen receptor $\mathrm{T}$ cells therapies against solid tumors. Cancer Lett (2017) 390:188-200. doi:10.1016/j.canlet.2016.12.037

38. Tal Y, Yaakobi S, Horovitz-Fried M, Safyon E, Rosental B, Porgador A, et al. An NCR1-based chimeric receptor endows T-cells with multiple anti-tumor specificities. Oncotarget (2014) 5:10949-58. doi:10.18632/oncotarget.1919

39. Zhang T, Wu MR, Sentman CL. An NKp30-based chimeric antigen receptor promotes $\mathrm{T}$ cell effector functions and antitumor efficacy in vivo. J Immunol (2012) 189:2290-9. doi:10.4049/jimmunol.1103495

40. Wargo JA, Robbins PF, Li Y, Zhao Y, El Gamil M, Caragacianu D, et al. Recognition of NY-ESO-1+ tumor cells by engineered lymphocytes is enhanced by improved vector design and epigenetic modulation of tumor antigen expression. Cancer Immunol Immunother (2009) 58:383-94. doi:10.1007/s00262-008-0562-X

41. Cohen H, Danin-Poleg Y, Cohen CJ, Sprecher E, Darvasi A, Kashi Y. Mono-nucleotide repeats (MNRs): a neglected polymorphism for generating high density genetic maps in silico. Hum Genet (2004) 115:213-20. doi:10.1007/s00439-004-1135-5

42. Ankri C, Cohen CJ. Out of the bitter came forth sweet: activating CD28dependent co-stimulation via PD-1 ligands. Oncoimmunology (2014) 3:e27399. doi:10.4161/onci.27399
43. Weizman E, Cohen CJ. Engineering T-cell specificity genetically to generate anti-melanoma reactivity. Methods Mol Biol (2016)1-9. doi:10.1007/ 7651_2015_297

44. Shamalov K, Tal Y, Ankri C, Cohen CJ. Adoptive T-cell immunotherapy: perfecting self-defenses. In: Klink M, editor. Interaction of Immune and Cancer Cells. Vienna: Springer (2014). p. 207-32.

45. Shamalov K, Levy SN, Horovitz-Fried M, Cohen CJ. The mutational status of p53 can influence its recognition by human T-cells. Oncoimmunology (2017) 6:e1285990. doi:10.1080/2162402X.2017.1285990

46. Wilde S, Sommermeyer D, Leisegang M, Frankenberger B, Mosetter B, Uckert W, et al. Human antitumor CD8+ T cells producing Th1 polycytokines show superior antigen sensitivity and tumor recognition. J Immunol (2012) 189:598-605. doi:10.4049/jimmunol.1102165

47. Kreiter S, Vormehr M, van de Roemer N, Diken M, Lower M, Diekmann J, et al. Mutant MHC class II epitopes drive therapeutic immune responses to cancer. Nature (2015) 520:692-6. doi:10.1038/nature14426

48. Haabeth OA, Tveita AA, Fauskanger M, Schjesvold F, Lorvik KB, Hofgaard PO, et al. How do CD4(+) T cells detect and eliminate tumor cells that either lack or express MHC class II molecules? Front Immunol (2014) 5:174. doi:10.3389/ fimmu.2014.00174

49. Zhang Y, Yan W, Mathew E, Bednar F, Wan S, Collins MA, et al. CD4+ $\mathrm{T}$ lymphocyte ablation prevents pancreatic carcinogenesis in mice. Cancer Immunol Res (2014) 2:423-35. doi:10.1158/2326-6066.CIR-14-0016-T

50. Gilham DE, Debets R, Pule M, Hawkins RE, Abken H. CAR-T cells and solid tumors: tuning T cells to challenge an inveterate foe. Trends Mol Med (2012) 18:377-84. doi:10.1016/j.molmed.2012.04.009

51. Cartellieri M, Bachmann M, Feldmann A, Bippes C, Stamova S, Wehner R, et al. Chimeric antigen receptor-engineered $\mathrm{T}$ cells for immunotherapy of cancer. J Biomed Biotechnol (2010) 2010:956304. doi:10.1155/2010/956304

52. Song DG, Ye Q, Poussin M, Harms GM, Figini M, Powell DJ Jr. CD27 costimulation augments the survival and antitumor activity of redirected human T cells in vivo. Blood (2012) 119:696-706. doi:10.1182/ blood-2011-03-344275

53. Kawalekar OU, O'Connor RS, Fraietta JA, Guo L, McGettigan SE, Posey AD Jr, et al. Distinct signaling of coreceptors regulates specific metabolism pathways and impacts memory development in CAR T cells. Immunity (2016) 44:380-90. doi:10.1016/j.immuni.2016.01.021

54. Liu X, Jiang S, Fang C, Li H, Zhang X, Zhang F, et al. Novel T cells with improved in vivo anti-tumor activity generated by RNA electroporation. Protein Cell (2017) 8(7):514-26. doi:10.1007/s13238-017-0422-6

55. Daniel-Meshulam I, Horovitz-Fried M, Cohen CJ. Enhanced antitumor activity mediated by human 4-1BB-engineered T cells. Int J Cancer (2013) 133:2903-13. doi:10.1002/ijc.28320

56. Hombach AA, Heiders J, Foppe M, Chmielewski M, Abken H. OX40 costimulation by a chimeric antigen receptor abrogates CD28 and IL-2 induced IL-10 secretion by redirected CD4(+) T cells. Oncoimmunology (2012) 1:458-66. doi:10.4161/onci.19855

57. Sentman CL, Barber MA, Barber A, Zhang T. NK cell receptors as tools in cancer immunotherapy. Adv Cancer Res (2006) 95:249-92. doi:10.1016/ S0065-230X(06)95007-6

58. Barber A, Rynda A, Sentman CL. Chimeric NKG2D expressing T cells eliminate immunosuppression and activate immunity within the ovarian tumor microenvironment. J Immunol (2009) 183:6939-47. doi:10.4049/ jimmunol.0902000

59. Barber A, Meehan KR, Sentman CL. Treatment of multiple myeloma with adoptively transferred chimeric NKG2D receptor-expressing T cells. Gene Ther (2011) 18:509-16. doi:10.1038/gt.2010.174

60. Marcu-Malina V, Heijhuurs S, van Buuren M, Hartkamp L, Strand S, Sebestyen $Z$, et al. Redirecting alphabeta $T$ cells against cancer cells by transfer of a broadly tumor-reactive gammadeltaT-cell receptor. Blood (2011) 118:50-9. doi:10.1182/blood-2010-12-325993

61. Morgan RA. Live and let die: a new suicide gene therapy moves to the clinic. Mol Ther (2012) 20:11-3. doi:10.1038/mt.2011.273

62. Muranski P, Restifo NP. Adoptive immunotherapy of cancer using CD4(+) T cells. Curr Opin Immunol (2009) 21:200-8. doi:10.1016/ j.coi.2009.02.004

63. Tran E, Turcotte S, Gros A, Robbins PF, Lu YC, Dudley ME, et al. Cancer immunotherapy based on mutation-specific CD4+ T cells in a patient with epithelial cancer. Science (2014) 344:641-5. doi:10.1126/science.1251102 
64. Cohen CJ, Zheng Z, Bray R, Zhao Y, Sherman LA, Rosenberg SA, et al. Recognition of fresh human tumor by human peripheral blood lymphocytes transduced with a bicistronic retroviral vector encoding a murine anti-p53 TCR. J Immunol (2005) 175:5799-808. doi:10.4049/jimmunol.175.9.5799

65. Hudspeth K, Silva-Santos B, Mavilio D. Natural cytotoxicity receptors: broader expression patterns and functions in innate and adaptive immune cells. Front Immunol (2013) 4:69. doi:10.3389/fimmu.2013.00069

66. Esin S, Batoni G, Counoupas C, Stringaro A, Brancatisano FL, Colone M, et al. Direct binding of human NK cell natural cytotoxicity receptor NKp44 to the surfaces of mycobacteria and other bacteria. Infect Immun (2008) 76:1719-27. doi:10.1128/IAI.00870-07
Conflict of Interest Statement: The authors declare that the research was conducted in the absence of any commercial or financial relationships that could be construed as a potential conflict of interest.

Copyright (c) 2017 Eisenberg, Shamalov, Meir, Hoogi, Sarkar, Pinker, Markel, Porgador and Cohen. This is an open-access article distributed under the terms of the Creative Commons Attribution License (CC BY). The use, distribution or reproduction in other forums is permitted, provided the original author(s) or licensor are credited and that the original publication in this journal is cited, in accordance with accepted academic practice. No use, distribution or reproduction is permitted which does not comply with these terms. 